\title{
Secondary resistance to bromocriptine and cabergoline therapy in hyperprolactinemia: One case of pregnancy with IVF-ET
}

\author{
Linlin Liang, Lu Wang, Qiaohua He, Cuilian Zhang and Hangsheng Li* \\ Reproductive Medical Center, the Affiliated People's Hospital of Zhengzhou University, Zhengzhou, China
}

\begin{abstract}
Background: For patients with hyperprolactinemia, primary dopamine agonist resistance occurs in 10-15\%, secondary resistance following initial biochemical and anti-proliferative response is very rare and has only been described in five previous cases. For women of childbearing age, hyperprolactinemia affects follicular development, potentially causing infertility. Currently, reports on pregnancy outcomes of secondary dopamine resistance in patients with unexplained hyperprolactinemia are scarce. In this paper, we report one case of pregnancy with unexplained bromocriptine- and cabergoline-resistant hyperprolactinemia, with follow-up data for four years after birth.

Case report: The 32-year-old patient had been diagnosed with primary infertility for four years. She has been treated with dopamine agonist therapy for 3 years. At first, she was initially treated with bromocriptine, marked reduction in prolactin concentration. After one years her prolactin concentration began to rise despite escalating doses of bromocriptine up to six tablets/day and cabergoline up $6 \mathrm{mg}$ weekly(two tablets each time, three times per week). Hypothalamic-pituitary lesions and other lesions were ruled out. After ovulation induction, the development and ovulation of dominant follicles were observed only occasionally. The patient failed to become pregnant after intrauterine insemination (IUI). However, after one cycle of IVF-ET(in vitro fertilization-embryo transfer)the patient became pregnant. She delivered a baby girl at full term, and the baby was normal over the course of the four-year follow-up.
\end{abstract}

Conclusion: While the mechanism of secondary resistance remains unknown and not possible to predict, the woman can become pregnant after IVF-ET, even if the PRL level remains higher than normal.

\begin{abstract}
Abbreviations: PRL-Prolactin; IUI-Intrauterine Insemination; IVF-ET-In Vitro Fertilization-Embryo Transfer.

\section{Introduction}

Prolactin (PRL) is an important hormone involved in regulating the gonadal axis. Hyperprolactinemia is a syndrome of hypothalamicpituitary axis reproductive and endocrine disorders that is associated with many causes and is characterized by high serum prolactin (PRL) levels and related clinical manifestations. Hyperprolactinemia involves the reproductive, endocrine, and nervous systems and is common in clinical practice. The incidence rate of hyperprolactinemia is $23.9 / 100$ 000 each year in 25- to 34-year-old women. Hyperprolactinemia causes gonadotropin deficiency and thus hypogonadism, resulting in menstrual cycle disorders, infertility, and/or galactorrhea [1]. In most cases, a dopamine agonist is used as a first-line treatment for most types of prolactinoma to reduce the PRL level and tumor size [2]; $80 \%$ to $90 \%$ of patients resume ovulation after treatment [3]. However, for some prolactinoma patients, the PRL level remains high with no tumor shrinkage after treatment with a high-dose of dopamine agonist, suggesting dopamine resistance [4]. A dopamine agonist is usually effective for treating idiopathic hyperprolactinemia [5]. In clinical practice, we treated one patient with unexplained hyperprolactinemia who was resistant to both bromocriptine and cabergoline. At first, she was initially treated with bromocriptine, marked reduction in prolactin concentration. After one years her prolactin concentration began to rise despite escalating doses of bromocriptine up to six tablets/day and cabergoline up $6 \mathrm{mg}$ weekly(two tablets each time, three times per
\end{abstract}

week). The patient failed to become ovulation induction and IUI. She finally got pregnancy after IVF-ET.; Her baby was followed up for four years after birth. In this paper, we report case data from the patient and perform a literature review of related cases.

\section{History of illness}

\section{Patient's basic history of illness}

The patient was female, 32 years old, with a body mass index (BMI) of 17. In April 2010, she visited our hospital for the "inability to become pregnant after being married for four years without the use of birth control methods." An inquiry into her detailed history revealed that her menstrual cycle started at 14 , with irregular menstruation that required medication for regulation. Tubal examination showed patent tubes. Semen test results of her husband were normal.

\section{Endocrine results and hyperprolactinemia-related tests}

A previous D3 hormone test at a local hospital showed a PRL level of $300 \mathrm{ng} / \mathrm{mL}$; after treatment with bromocriptine, four tablets/day,

${ }^{\star}$ Correspondence to: Hangsheng Li, Reproductive Medical Center, the Affiliated People's Hospital of Zhengzhou University, Zhengzhou, China, Tel: +8613523585116; E-mail: xiaohongfan0617@126.com

Key words: dopamine agonist resistance, hyperprolactinemia, pregnancy

Received: May 21, 2019; Accepted: May 28, 2019; Published: May 30, 2019 
her PRL level can reduce to $30 \mathrm{ng} / \mathrm{mL}$. After one years her prolactin concentration began to rise despite escalating doses of bromocriptine up to six tablets/day Therefore, the patient's treatment was changed to cabergoline, two tablets/day, PO, three times per week. After treatment, a sex hormone test revealed a follicle-stimulating hormone (FSH) level of $2.13 \mathrm{mIU} / \mathrm{mL}$, a luteinizing hormone ( $\mathrm{LH})$ level of $0.52 \mathrm{mIU} / \mathrm{mL}$, a PRL level of $115.2 \mathrm{ng} / \mathrm{mL}$, a progesterone (P) level of $0.98 \mathrm{ng} / \mathrm{mL}$, and an estradiol (E2) level of $9.14 \mathrm{pg} / \mathrm{mL}$. Brain computed tomography and magnetic resonance imaging (per a neurosurgeon's recommendation) were normal. The patient's thyroid and adrenal glands were normal, and her visual field was normal. The patient complained of severe galactorrhea, with no significant growth of body hair. After treatment with a higher dose of cabergoline, the patient reported intolerable nausea, vomiting, and worsening dizziness, so the dose was reduced to the original level.

\section{Ovulation induction}

Repeated ultrasound B exams at a local hospital revealed no visible development or ovulation of dominant follicles. Given her irregular menstruation, the patient was pre-treated with medications (progynova + progesterone) to induce two artificial cycles, and she continued to take cabergoline. After two cycles of treatment, her PRL was $105.7 \mathrm{ng} / \mathrm{mL}$. With patient consent, $50 \mathrm{mg}$ of clomiphene citrate was given to induce ovulation for two cycles (day five of the menstrual cycle, one capsule, PO, QD). During cycle one, dominant follicles were developed to $19.4 \mathrm{~mm}$, and the endometrial thickness was $7.1 \mathrm{~mm} /$ type B. Hormone tests returned a serum E2 level of $473.914 \mathrm{pg} / \mathrm{mL}$, an $\mathrm{LH}$ level of $15.2 \mathrm{mIU} / \mathrm{mL}$, a P level of $0.61 \mathrm{ng} / \mathrm{mL}$, and a PRL level of 99.01 $\mathrm{ng} / \mathrm{mL}$. The patient was given an intramuscular (IM) dose (10 $000 \mathrm{IU})$ of human chorionic gonadotropin (HCG) and was then instructed to have sexual intercourse. Luteal support was given after follicle rupture. Fourteen days later, the serum $\beta$-HCG level indicated that the patient had failed to become pregnant. During cycle two, dominant follicles were developed to $18.9 \mathrm{~mm}$, and the endometrial thickness was 6.7 $\mathrm{mm} /$ type B. Hormone tests returned a serum E2 level of $403.4 \mathrm{pg} /$ $\mathrm{mL}$, an LH level of $16.8 \mathrm{mIU} / \mathrm{mL}$, a P level of $0.52 \mathrm{ng} / \mathrm{mL}$, and a PRL level of $117.9 \mathrm{ng} / \mathrm{mL}$. The patient was given an IM dose (10 $000 \mathrm{IU})$ of HCG and was then instructed to have sexual intercourse. Ultrasound $\mathrm{B}$ showed that the follicles luteinized but did not rupture. During cycle three, human menopausal gonadotropin (hMG) was given to induce ovulation (day three of the menstrual cycle, $75 \mathrm{IU}$, every other day, for a total of five injections). Dominant follicles were developed to 19.8 $\mathrm{mm}$, and the endometrial thickness was $8.8 \mathrm{~mm} /$ type B. Hormone tests returned a serum E2 level of $313.6 \mathrm{pg} / \mathrm{mL}$, an $\mathrm{LH}$ level of $12.8 \mathrm{mIU} / \mathrm{mL}$, a P level of $0.47 \mathrm{ng} / \mathrm{mL}$, and a PRL level of $107.81 \mathrm{ng} / \mathrm{mL}$. The patient was given an IM dose (10 $000 \mathrm{IU})$ of HCG and was then instructed to have sexual intercourse. Luteal support was given after follicle rupture. Fourteen days later, the serum $\beta$-HCG level indicated that the patient had failed to become pregnant. During cycle four, hMG was given to induce ovulation (day three of the menstrual cycle, $75 \mathrm{IU}$, for a total of 12 injections). No obvious development of dominant follicles was observed.

\section{Intrauterine insemination (IUI)}

The patient insisted on receiving assisted reproductive treatment. Given her condition, IUI was recommended. After treatment to induce two artificial cycles, a higher dose of hMG was given to induce ovulation. During cycle one, the development and ovulation of dominant follicles was observed, and IUI and luteal support were given. However, the patient failed to become pregnant. During cycles two and three, hMG was given to induce ovulation, and an IM dose (10 000 IU) of HCG was then given after the development of dominant follicles. However, the follicles luteinized but did not rupture.

\section{IVF-ET (In vitro fertilization-embryo transfer)}

We advised the patient to reduce her mental stress and planned to perform IUI after additional ovulation induction. The patient declined additional IUI and insisted on receiving IVF. We developed a plan with a longer timeline. After the induction of artificial cycles, gonadotropin-releasing hormone agonist (GnRHa; triptorelin) was given on day 25 of the menstrual cycle $(0.1 \mathrm{mg}$, subcutaneous injection, for 18 days). Hormone tests returned a serum E2 level of $9.14 \mathrm{pg} / \mathrm{mL}$, an LH level of $0.52 \mathrm{mIU} / \mathrm{mL}$, a P level of $0.98 \mathrm{ng} / \mathrm{mL}$, and a PRL level of $115.2 \mathrm{ng} / \mathrm{mL}$. Then, $150 \mathrm{IU}$ of $\beta$-FSH was given for 12 days to induce ovulation. By the time of HCG dosing, eight follicles were larger than $14 \mathrm{~mm}$. Hormone tests returned a serum E2 level of $2,568 \mathrm{pg} / \mathrm{mL}$, an $\mathrm{LH}$ level of $0.58 \mathrm{mIU} / \mathrm{mL}$, a P level of $1.71 \mathrm{ng} / \mathrm{mL}$, and a PRL level of $171.2 \mathrm{ng} / \mathrm{mL}$. HCG (8000 IU) was given to induce ovulation, and eggs were collected 36 hours and 25 minutes later. A total of 10 eggs were collected, of which seven were in metaphase II (MII); five were fertilized, and five embryos were considered viable (4c, $5 \%$ fragments; $8 \mathrm{c}, 10 \%$ fragments; $6 \mathrm{c}, 10 \%$ fragments; $8 \mathrm{c}, 20 \%$ fragments; 9c, 20\% fragments). Two considered viable embryos were implanted on day three after egg collection. Luteal phase support was given on the day of embryo transfer. Additional embryos considered viable according to morphologic criteria were frozen for later transfer in subsequent frozen embryo transfer (FET) cycles.

\section{Pregancy follow-up}

$\beta$-HCG was measured 2 weeks after embryo transfer, the number is $420 \mathrm{pg} / \mathrm{ml}$. Then at gestational week $7-9$, fetal heart beating can be seen by ultrasonography. Luteal support was decreased untill 90 days, and her cabergoline dose was tapered from gestation month three until withdrawal. In August 2012, she delivered a baby girl at full term. The baby weighed $3.4 \mathrm{~kg}$, with no maternal or newborn complications. She produced enough milk during breast-feeding, and her menstrual cycle resumed at month seven after birth. At the time, her menstrual cycle was 30 to 40 days, with moderate flow. The patient still had galactorrhea after the end of breast-feeding. She had a serum PRL test again at Octorber, 2016, and the PRL is $45 \mathrm{ng} / \mathrm{ml}$.She did not take other drugs after dopamine agonist withdrawal. She did not use any birth control methods nor did she become pregnant during the four-year follow-up period.

\section{Newborn follow-up}

The baby girl underwent normal development, and her height, weight, blood pressure, head circumference, and chest circumference were similar to those of other children her age. Her motor coordination and mental development were normal. Her social skills (per the Achenbach Child Behavior Checklist [CBCL] score) were similar to those of children of her age.

\section{Discussion}

We describe a very rare case of a 32-year-old woman with unexplained hyperprolactinemia, who following 1 year of almost complete anti-secretory response dopamine agonist therapy first with bromocriptine, developed secondary resistance to bromocriptine and cabergoline. This phenomenon has hitherto been described scarce. 
PRL is secreted by prolactin cells in the anterior pituitary; however, recent studies have shown that PRL may also be secreted by other types of cells in the anterior pituitary [6]. PRL plays an important role during breast-feeding and pregnancy and is involved in certain physiological functions, such as angiogenesis, immune regulation, and osmotic regulation [7]. Under physiological conditions, PRL regulation is predominantly inhibitory, and any factor that interferes with hypothalamic dopamine synthesis and its transport to the pituitary or affects the interaction between dopamine and its receptors can weaken the inhibitory regulation, resulting in hyperprolactinemia. Common causes include physiological, pathological, pharmacological, and idiopathic factors. Idiopathic hyperprolactinemia is defined as high serum PRL (typically $<4.55 \mathrm{nmol} / \mathrm{L}$ ), with negative examination results of the pituitary and central nervous systems and positive symptoms including galactorrhea, oligomenorrhea, and amenorrhea.

For the patient in this report, physiological factors were ruled out. Moreover, the patient had no history of long-term use of other drugs. Physical examination and laboratory testing ruled out obvious pathological changes, such as hypothalamic-pituitary diseases. Thus, the patient was diagnosed as having idiopathic hyperprolactinemia. However, most patients with idiopathic hyperprolactinemia have relatively low PRL levels, whereas this patient's baseline PRL was $>300$ $\mathrm{pg} / \mathrm{mL}$; the chance of prolactinoma is greater if the PRL level is $>250$ $\mathrm{pg} / \mathrm{mL}$ [8]. For this patient, hyperprolactinemia might be related to micro-prolactinoma, which is undetectable with available diagnostic tools. Another potential factor is hypothalamic regulatory disorders [9].

Dopamine receptor agonists (such as cabergoline and bromocriptine) are current common, first-line treatments for hyperprolactinemia. Cabergoline is a relatively new specific dopamine D2 receptor agonist. Triantafilo et al. [10] conducted a meta-analysis and found that cabergoline is more effective for amenorrhea, irregular menstruation, and galactorrhea; improves pregnancy rates; and is more advantageous in patients unable to tolerate bromocriptine or who are resistant to bromocriptine.

The patient's medical records showed that her PRL level was still high after bromocriptine treatment (six tablets/day)after one-year treated with bromocriptine. She was then converted to cabergoline with a dose escalation to the maximum tolerated dose, but her PRL level remained high. According to the literature, dopamine agonist resistance is defined as higher than normal serum PRL and/or no tumor shrinkage after dopamine treatment. Up to $25 \%$ of patients fail to achieve normal PRL levels after bromocriptine treatment, and $10 \%$ to $15 \%$ of patients fail to have their PRL levels under control after cabergoline treatment. After bromocriptine treatment, $20 \%$ of patients with micro-prolactinoma and $30 \%$ of patients with macroprolactinoma continue to have high PRL levels, but cabergoline can increase the response rates by $10 \%$ and $20 \%$, respectively, likely related to the higher affinity of cabergoline to dopamine-binding sites, the longer duration of binding, and the slower clearance [11]. Nevertheless, one study showed that two cabergoline-resistant prolactinoma patients achieved satisfactory treatment results after bromocriptine treatment [12].

Dopamine agonist resistance is uncommon in prolactinoma patients; cabergoline $2.0 \mathrm{mg} /$ week is usually effective for controlling PRL secretion and reducing prolactinoma size [13]. Few patients who initially respond to dopamine agonists develop later resistance; to date, only five such cases have been reported, and the mechanism of secondary resistance is unknown [14]. In this report, the patient had hyperprolactinemia, and her serum PRL level remained uncontrolled after one-year treated with bromocriptine. which can be diagnosised secondary resistance?

For patients with dopamine agonist resistantance, treatment options include conversion to other dopamine agonists and a dose increase to the maximum tolerated dose. For patients unable to tolerate oral bromocriptine, the drug may be given via the vagina. Ono et al [15] showed that among 60 treatment-naive prolactinoma patients, 11 (18.3\%) required a dose of $>2 \mathrm{mg} /$ week cabergoline to achieve normal PRL levels (in one case, the dose even reached $11 \mathrm{mg} /$ week). In this report, the patient's PRL level remained uncontrolled after conversion; it is unknown whether a higher cabergoline dose could achieve better results since the patient complained of severe adverse reactions if the cabergoline dose was greater than $3 \mathrm{mg} /$ week. Therefore, further research is needed to investigate how to control hyperprolactinemia while mitigating adverse reactions.

In this report, the patient visited our hospitals mainly for infertility (caused by hyperprolactinemia). Numerous studies have shown that hyperprolactinemia is a common cause of anovulation, as hyperprolactinemia can act on the hypothalamus via short-loop feedback to stimulate dopamine secretion, which, in turn, inhibits GnRH, FSH, and LH secretion [16] and reduces kisspeptin expression [17], thereby inhibiting follicle development, maturation, and ovulation and inducing low egg quality, low fertilizing capability, division disorder, and infertility. Inhibition of LH secretion causes luteal degradation, luteal dysfunction, a high spontaneous abortion rate, and a high risk of infertility.

For patients who wish to become pregnant, ovulation induction therapy, such as clomiphene citrate and gonadotropin, may be given [18]. In this report, the patient received ovulation induction therapy. However, during treatment, it was noted that follicle development and ovulation occurred only during cycle one of either drug treatment. During clomiphene citrate treatment, dominant follicles luteinized during cycle two; during hMG treatment, no dominant follicle growth was observed during cycle two; during hMG treatment with a higher dose, follicles luteinized during cycles two and three. During IVF-ET, the embryo quality was not high, and the PRL level increased with E2. Follicular dysplasia may be related to mental stress and high PRL levels.

Studies have reached similar conclusions about the safety of cabergoline and bromocriptine in early pregnancy. To date, approximately 900 patients have taken cabergoline in early pregnancy, and safety evaluations of fetuses and newborns showed no significant increases in the early abortion, preterm birth, and multiple pregnancy rates or birth defects [19], which was consistent with the follow-up findings of this report. For this patient, after pregnancy was confirmed, the cabergoline dose was tapered until withdrawal. The baby girl was followed up for four years, and her height, weight, blood pressure, head circumference, and chest circumference were similar to those of children of the same age. Her motor coordination and mental development were normal, and her social skills (per CBCL score) were similar to those of children of the same age. For the patient, her menstrual cycle resumed at month seven after birth. At the time, her menstrual cycle was 30 to 40 days, with moderate flow. She still had galactorrhea after the end of breast-feeding. She did not take other drugs after dopamine agonist withdrawal. She did not use birth control methods nor did she become pregnant during the four-year follow-up period. She have a serum PRL test in 10, 2016 and the PRL is $45 \mathrm{ng} / \mathrm{ml}$. A 35 -year follow-up study showed that for hyperprolactinemia patients, treatment was highly 
effective in $20.8 \%(10 / 48)$ of the patients and effective in $68.8 \%(33 / 48)$ of the patients. Higher cabergoline doses were required in only $10.4 \%$ (5/48) of the patients due to increasing PRL levels [20]. Thus, highly effective treatment is available for hyperprolactinemia, but PRL levels should be monitored regularly, and hypothalamic-pituitary diseases must be ruled out in cases of increasing PRL levels.

\section{Conclusion}

We described a rare case of a 32-year-old woman with unexplained hyperprolactinemia who developed secondary dopamine agonist resistance following 1 years of DA therapy. As the underlying mechanism is unknown, patients who wish to become pregnant should be advised to reduce mental stress, and treatment may be given to reduce PRL levels to restore ovulation. Ovulation induction and assisted reproductive therapy may be given as necessary to facilitate pregnancy. Once the patient becomes pregnant, the dose of the dopamine agonist should be tapered as soon as possible to minimize its effect on the fetus.

\section{Acknowledgements}

We are grateful to Mrs. wang for her history illness and to the nurses and staff of the reproductive center of Henan Province People,s Hospital, Zhengzhou, Henan.

\section{Availability of data and materials}

The datasets used and/or analyzed during the current study available from the corresponding author on reasonable request.

\section{Authors' contributions}

LinlinLiang,Ph,D: Manuscript Drafting, Critical Discussion;

Lu Wang,MD: Execution

QiaohuaHe,Ph,D: Execution;

CuilianZhang,Ph,D: Study Guide,

HangshengLi,MD :Manuscript Drafting, Critical Discussion.

All authors read and approved the final manuscript.

\section{Competing interests}

The authors declare that they have no competing interests.

\section{Consent for publication}

Not applicable.

\section{Author details}

\section{Author affiliations}

7th Weiwu Road, Reproductive Medical Center, Henan Provincial People's Hospital, Zhengzhou, China and Reproductive Medical Center, the Affiliated People's Hospital of Zhengzhou University, Zhengzhou, China

\section{References}

1. Saejong R, Dangrat C, Techatrisak K1, Angsuwatthana S1, Rattanachaiyanont M1, et al. (2013) Hyperprolactinemia: a 12-year retrospective study at gynecologic endocrinology unit, Siriraj Hospital. J Med Assoc Thai 96: 1247-1256. [Crossref]

2. Klibanski A (2010) Clinical practice. Prolactinomas. N Engl J Med 36: 1219-1226. [Crossref]

3. Cecenarro LA, Estario P, Estario ME, Fux-Otta C (2015) Update on endocrinology: management of prolactinomas during pregnancy. Rev Fac Cien Med Univ Nac Cordoba 72: 170-174. [Crossref]

4. Molitch ME (2005) Pharmacologic resistance in prolactinoma patients. Pituitary 8: 4352. [Crossref]

5. Chen H, Fu J, Huang W (2016) Dopamine agonists for preventing future miscarriage in women with idiopathic hyperprolactinemia and recurrent miscarriage history. Cochrane Database Syst Rev 7: CD008883. [Crossref]

6. Ignacak A, Kasztelnik M, Sliwa T, Korbut RA, Rajda K, et al. (2012) Prolactin--not only lactotrophin. A "new" view of the "old" hormone. J Physiol Pharmacol 63: 435443. [Crossref]

7. Pontecorvi A, Lello S (2015) Hyperprolactinemia: pathophysiology and therapeutic approach. Gynecol Endocrinol 31: 506-510. [Crossref]

8. Paepegaey AC, Veron L, Wimmer MC, Christin-Maitre S (2016) Misleading diagnosis of hyperprolactinemia in women. Gynecol Obstet Fertil 44: 181-186. [Crossref]

9. Martin TL, Kim M, Malarkey WB (1985) The natural history of idiopathic hyperprolactinemia. J Clin Endocrinol Metab 60: 855-858. [Crossref]

10. Triantafilo N, Castro-Gutiérrez V, Rada G (2016) Cabergoline or bromocriptine for prolactinoma? Medwave 16: e6545.

11. Colao A, DiSarno A, Sarnacchiaro F (1997) Prolactinomas resistant to standard dopamine agonists respond to chronic cabergoline treatment. J Clin Endocrinol Metab 82: 876-883. [Crossref]

12. Iyer P, Molitch ME (2011) Positive prolactin response to bromocriptine in 2 patients with cabergoline-resistant prolactinomas. Endocr Pract 17: e55-e58. [Crossref]

13. Vroonen L, Jaffrain-Rea ML, Petrossians P (2012) Prolactinomas resistant to standard doses of cabergoline: a multicenter study of 92 patients. Eur J Endocrinol 167: 651662. [Crossref]

14. Behan LA, Draman MS, Moran C, King T, Crowley RK, et al. (2011) Secondary resistance to cabergoline therapy in a macroprolactinoma: a case report and literature review. Pituitary 14: 362-366. [Crossref]

15. Ono M, Miki N, Kawamata T (2008) Prospective study of high-dose cabergoline treatment of prolactinomas in 150 patients. J Clin Endocrinol Metab 93: 4721-4727. [Crossref]

16. Kaiser UB (2012) Hyperprolactinemia and infertility: new insights. $J$ Clin Invest 122 3467-3468. [Crossref]

17. Sonigo C, Bouilly J, Carré N, Tolle V, Caraty A, et al. (2012) Hyperprolactinemiainduced ovarian acyclicity is reversed by kisspeptin administration. J Clin Invest 122 : 3791-3795. [Crossref]

18. Molitch ME (2014) Management of medically refractory prolactinoma. J Neurooncol 117: 421-428. [Crossref]

19. Maiter D (2016) Prolactinoma and pregnancy: From the wish of conception to lactation. Ann Endocrinol (Paris) 77: 128-134. [Crossref]

20. Képénékian L, Cebula H, Castinetti F, Graillon T, Brue T, et al. (2016) Long-term outcome of macroprolactinomas. Ann Endocrinol (Paris) 77: 641-648. [Crossref]

Copyright: (C2019 Liang L. This is an open-access article distributed under the terms of the Creative Commons Attribution License, which permits unrestricted use, distribution, and reproduction in any medium, provided the original author and source are credited. 